\title{
O transformador: teoria, construção e análise do rendimento
}

The transformer: theory, construction and analysis of performance

\author{
André Luíz Alves ${ }^{*} @$, Estevão Modolo de Souza ${ }^{2}$, Cledrlon Pereira Machado ${ }^{1}$, \\ Kaio Alan Littike ${ }^{1}$, Deywindir Michell Santana da Silva ${ }^{1}$ \\ ${ }^{1}$ Universidade Federal do Espírito Santo, São Mateus, ES, Brasil. \\ ${ }^{2}$ Instituto Federal do Espírito Santo Brasil, São Mateus, ES, Brasil.
}

Recebido em 25 de novembro de 2021. Revisado em 14 de dezembro de 2021. Aceito em 30 de dezembro de 2021.

Neste trabalho foi apresentado de forma detalhada as etapas da construção de um transformador do tipo nuclear e como medidas elétricas podem ser utilizadas para se extrair parâmetros de relevância dele. Descreveu-se de forma detalhada a teoria física para o modelo de um transformador real. Teoricamente, foi concluído que este pode ser tratado como um transformador ideal inserido em um circuito equivalente com impedâncias. Nas análises experimentais foi possível obter as resistências e reatâncias das bobinas e do núcleo e com isto estimar o rendimento do transformador.

Palavras-chave: Experiência, Transformador, Circuito Equivalente, Potências, Rendimento.

In this work, the stages of construction of a nuclear-type transformer were presented in detail and how electrical measurements can be used to extract relevant parameters from it. The physical theory for the model of a real transformer has been briefly described. Theoretically, it was concluded that this can be treated as an ideal transformer plus external impedances. In the experimental analyses, it was possible to obtain the resistances and reactances of the coils and the core and with this estimate the transformer efficiency.

Keywords: Experience, Transformer, Equivalent Circuit, Powers, Performance.

\section{Introdução}

O uso de sistemas físicos reais como recurso didático de caráter teórico-experimental, potencializa o diálogo em sala de aula, aproxima o estudante da realidade que o cerca e tem a função pedagógica de propiciar uma melhor compreensão conceituada de teorias físicas [1]. Tendo em vista que o ensino da eletricidade e eletromagnetismo é uma das áreas em que há maior abstração das grandezas físicas associadas e uma das que os estudantes apresentam maiores dificuldades de aprendizado, fazse necessário aumentar a motivação deles utilizando-se, dentre vários métodos, as práticas experimentais [1, 2]. É importante, por meio destas práticas, conscientizar o estudante de que o eletromagnetismo é a ciência básica de grande parte da tecnologia moderna em que vivemos e contribui com a qualidade de vida de toda uma população.

A energia elétrica com Corrente Alternada (CA) é primordial no funcionamento de diversas máquinas elétricas em nossos cotidianos. Por meio de fios, que se estendem por milhares de quilômetros, esta chega de forma segura nas residências levando conforto e praticidade por meio dos equipamentos elétricos e eletrônicos. Nas

\footnotetext{
*Endereço de correspondência: andre.alves@ufes.br
}

indústrias movem máquinas de altas potências, essenciais na produção e no desenvolvimento de uma região. $\mathrm{O}$ transporte com longos alcances sem perdas consideráveis não seria possível sem o uso do transformador. A energia elétrica após ser produzida em uma estação geradora, tem seu valor de tensão consideravelmente aumentado, o que permite uma reduzida corrente nos fios condutores durante seu transporte, diminuindo as perdas por efeito Joule [3].

Geralmente ao se abordar o transformador nos laboratórios de ensino de eletromagnetismo, nas instituições de curso superior, tem-se dado pouca ênfase à transferências de potências, rendimentos e os parâmetros elétricos que podem ser obtidos das tensões, correntes e potências nas bobinas do primário e secundário. Muitas vezes, são tratados os princípios básicos experimentais de funcionamento e a relação entre as tensões com os números de espiras nas bobinas.

Neste trabalho é proposto investigar o efeito de um transformador sobre a tensão, corrente e energia do tipo CA. Foi realizada uma descrição teórica, baseada no livro Máquinas Elétricas de autoria de Stephen D. Umans, 2014 [4, para uma discussão das grandezas física de um transformador que consomem energia elétrica. Estas grandezas, que estão presentes no núcleo ferromagnético e nas bobinas do transformador, influenciam no seu 
rendimento. O transformador que foi construído é do tipo nuclear (núcleo tipo "U"). Este é de fácil montagem e prático para uma abordagem experimental em um laboratório de ensino. Nos resultados experimentais, é demonstrado como os estudantes podem realizar medições de transformação de corrente e tensão entre as bobinas do transformador e analisar a transmissão de potência fornecida a uma carga resistiva. As práticas aqui desenvolvidas permitem que o estudante confronte teoria e experimento. Podem ser aplicadas no ensino técnico e superior.

\section{Aspectos Teóricos}

\subsection{O transformador}

Um transformador é um dispositivo que converte, por meio da ação de um campo magnético, a energia elétrica CA de uma dada frequência e tensão em energia elétrica CA de mesma frequência, mas outro nível de tensão [3]. De acordo com a ilustração da Figura 1(a) ele consiste em pelo menos dois enrolamentos (bobinas), em torno de um núcleo comum de material ferromagnético. As grandezas descritas nesta Figura serão discutidas na próxima seção. Na Figura 1(b) é mostrado o transformador didático produzido neste trabalho. Nele a bobina do secundário possui diversas derivações para se verificar a relação entre tensões e número de espiras. Em um transformador o enrolamento ligado à fonte de energia é denominado de bobina do primário e o enrolamento conectado às cargas é denominada de bobina do secundário [3, 5]. Por simplicidade utiliza-se em algumas situações apenas os termos primário e secundário. Não há conexão elétrica entre os fios das bobinas. A única conexão é devida ao fluxo magnético comum presente dentro do núcleo.

Em um transformador real existem perdas de energia por efeito Joule nas bobinas do primário e secundário, perdas por correntes de Foucault (correntes parasitas), histerese e pelo fluxo disperso nos enrolamentos [3]. Para se diminuir as correntes de Foucault o núcleo é construído de chapas delgadas de aço-Si (veja o núcleo nas Figuras 1(a) e (b)) de alta permeabilidade magnética

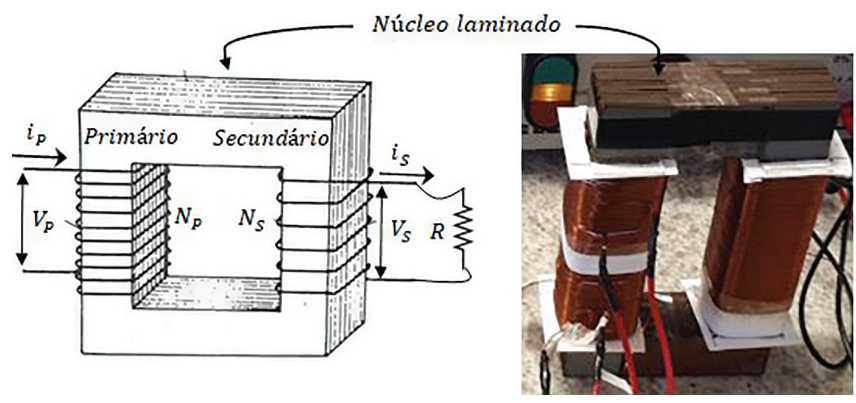

Figura 1: (a) llustração de um transformador nuclear (núcleo tipo "U"). Adaptado de Electrônica PT [5]. (b) O transformador construído pelos autores deste trabalho. e resistividade elétrica relativamente alta [5, 6]. Por sua vez, no transformador ideal todas as perdas são desconsideradas [6]. Nas descrições teóricas das próximas seções, tratam-se primeiro do modelo do transformador ideal, obtendo-se relações entre tensão, corrente e número de espiras entre as bobinas. Este modelo é utilizando, dentro de certas considerações, na descrição do modelo transformador real (veja a seção 2.3.

\subsection{O transformador ideal}

Suponha o caso em que o transformador da Figura 1(a) seja do tipo ideal. Quando a bobina do primário, $\operatorname{com} N_{P}$ espiras, é ligada a uma tensão alternada $V_{P}$, circulará nela uma corrente alternada $i_{P}$. Pela lei de Ampère, esta corrente produz um campo magnético que ao atravessar a área de seção reta do núcleo, produzirá um fluxo alternado $\Phi$. Através do núcleo ferromagnético, este fluxo irá atravessar as $N_{s}$ espiras do enrolamento do secundário produzindo, segundo a lei de Faraday, uma tensão $V_{S}$ e uma corrente alternada $i_{s}$, que alimentará um circuito; no caso da Figura 11(a) um resistor. De acordo com a lei de Faraday, a tensão nos terminais do primário é:

$$
V_{P}=N_{P} \frac{d \Phi}{d t}
$$

e no secundário:

$$
V_{S}=N_{S} \frac{d \Phi}{d t} .
$$

Dividindo a Equação (1) pela Equação (2), obtém-se:

$$
\frac{V_{P}}{V_{S}}=\frac{N_{P}}{N_{S}} .
$$

Desta forma, se $N_{P} / N_{S}>1$ tem-se que $V_{P}>V_{S}$, um transformador que diminui a tensão, e se $N_{P} / N_{S}<1$ tem-se que $V_{P}<V_{S}$, um transformador que aumenta a tensão [6].

Levando em consideração que em um transformador ideal não há perdas, então os ângulos de fase entre a tensão $\left(\theta_{v}\right)$ e a corrente $\left(\theta_{i}\right)$ no primário é igual à do secundário (veja o Apêndice I). Nessas considerações, pode-se escrever que para um transformador ideal a potência ativa fornecida ao primário é igual a potência ativa fornecida pelo secundário à sua carga (veja sobre o triângulo das potências no Apêndice II . Logo:

$$
V_{P} i_{P} \cos \theta=V_{S} i_{S} \cos \theta
$$

Fazendo a Equação (3) na Equação (4), obtém-se para a transformação de corrente:

$$
\frac{i_{P}}{i_{S}}=\frac{N_{S}}{N_{P}} .
$$

Portanto, um transformador ideal transforma correntes na razão inversa das espiras de seus enrolamentos. 
Outra forma de se obter a Equação (5) é levando em consideração o circuito magnético do transformador. O núcleo com comprimento médio, $l$, área de seção reta, $A$, e permeabilidade magnética, $\mu$, juntamente com as bobinas do primário e secundário formam um circuito magnético, análogo ao caso elétrico [7]. Para o transformador ideal da Figura 1, tem-se que:

$$
N_{P} i_{P}-N_{S} i_{S}=\frac{A}{\mu l} \Phi,
$$

no qual os termos $N_{P} i_{P}$ e $N_{S} i_{S}$ representam as forças magnetomotrizes $\left(\mathrm{fmm}^{\prime} \mathrm{s}\right)$ das bobinas do primário e secundário, respectivamente, $\mathcal{R}=A / \mu l$ é a relutância do núcleo e $\Phi$ o fluxo do campo magnético. A $\mathrm{fmm}$ desempenha um papel semelhante à força eletromotriz em um circuito elétrico. Ainda na Equação (6), $\mathcal{R}$ desempenha um papel semelhante a uma resistência elétrica e $\Phi$ é análogo à corrente elétrica. O sinal negativo da força $f m m$ para a bobina do secundário $\left(N_{S} i_{S}\right)$ é devido ao fato de a corrente no secundário tender a produzir um fluxo em sentido oposto ao fluxo produzido pelo primário, de acordo com a lei de indução de Faraday/Lenz [6, 7]. Em um transformador ideal, $\mu$ possui um valor suficientemente grande de tal forma que uma $\mathrm{fmm}$ resultante desprezível deve ser suficiente para geral o fluxo. Assim, na Equação (6):

$$
N_{P} i_{P}-N_{S} i_{S}=0
$$

reagrupando-se os termos dessa equação, reescreve-se a Equação (5) para a transformação de corrente.

Embora em um transformador a impedância $(Z)$ esteja ligada à bobina do secundário, é possível tratála como se esta estivesse ligada ao primário do transformador e o secundário em curto, em um circuito equivalente. Embora, no circuito real, não haja nenhum equipamento elétrico físico conectado ao primário do transformador, a consideração do circuito equivalente facilita a análise de potências quando se trata de um transformador real. Utiliza-se os termos transformar, refletir ou referenciar a impedância do secundário para o primário. Em nosso trabalho, a impedância utilizada foi, por simplicidade, uma carga resistiva. Esta carga, referenciada ao primário, será designada por $R_{P}$, de tal forma que:

$$
R_{P}=\frac{V_{P}}{i_{P}}
$$

Utilizando as Equações (3), (5) e (8) obtém-se para a transformação da resistência:

$$
R_{P}=\left(\frac{N_{P}}{N_{S}}\right)^{2} R_{S}
$$

Desta forma, a transformação de resistências ocorre com o quadrado da relação do número de espiras.

Na Figura 2(a) é mostrado o circuito convencional do transformador com a resistência, $R_{S}$, alimentada
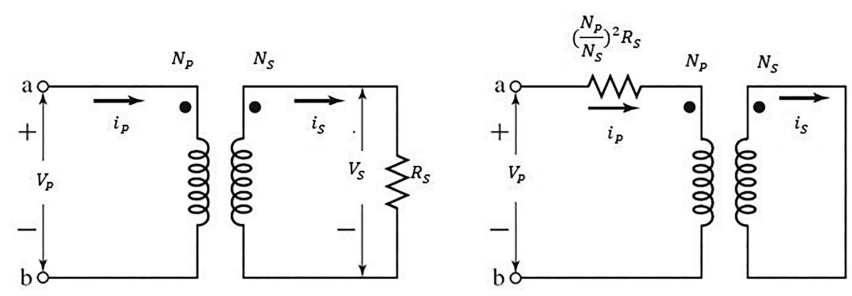

Figura 2: (a) Ilustração de um circuito de um transformador Ideal. (b) O circuito equivalente com a resistência do secundário referenciada ao primário. Adaptado de Stephen D. Umans, 2014 [4].

pela bobina do secundário. No circuito equivalente da Figura 2(b), o secundário encontra-se em curto e a resistência, $R_{S}$, é agora referenciada ao primário do transformador como $R_{P}$, de acordo com a Equação (9). Os círculos fechados representam pontos com a mesma polaridade da tensão nas bobinas.

Como exemplo, suponha que $V_{P}=30 \mathrm{~V}, N_{P} / N_{S}=$ $5: 1$ e $R=4 \Omega$. Para o circuito da Figura 2(a), obtémse da Equação (3) que $V_{S}=6 \mathrm{~V}$. Sabendo que $V_{S}=$ $R i_{S}$, tem-se que: $i_{S}=1,5 \mathrm{~A}$. Da Equação (5), obtém-se que $i_{P}=0,3 \mathrm{~A}$. A potência dissipada na resistência é $P=4 \Omega \cdot(1,5 A)^{2}=9 \mathrm{~W}$. Agora, considerando o circuito da Figura 2(b), tem-se da Equação (9) que $R_{P}=100$ $\Omega$. Para calcular $i_{P}$, pode-se fazer $i_{P}=30 \mathrm{~V} / 100 \Omega=$ $0,3 \mathrm{~A}$, e utilizando a Equação (5), obtém-se $i_{S}=1,5 \mathrm{~A}$. Estes valores são semelhantes ao obtido para o circuito da Figura 2(a). A potência dissipada neste circuito é $P=100 \Omega \cdot(0,3 \mathrm{~A})^{2}=9 \mathrm{~W}$. Portanto, os circuitos da Figura 2 são equivalentes.

\subsection{Transformador real e o circuito equivalente}

Em um transformador real devem ser levadas em consideração os efeitos dos fluxos dispersos, das resistências dos enrolamentos e das perdas no núcleo pelas correntes parasitas e histereses magnéticas. Nas próximas seções abordam-se cada um destes componentes e como eles serão incluídos em um circuito equivalente de um transformador real.

\subsubsection{Reatância de dispersão e resistência das bobinas}

Na Figura 3 está ilustrado um transformador, no qual os retângulos menores 1 e 2 representam as bobinas do primário e secundário. As linhas pontilhadas representam o campo magnético através do núcleo e os símbolos "•" e "x" o sentido da corrente, saindo e entrando, respectivamente, em cada bobina.

O fluxo que atravessa o enrolamento do primário pode ser dividido em duas componentes: o fluxo mútuo resultante $\left(\Phi_{M}\right)$, confinado essencialmente ao núcleo de ferro e produzido pelo efeito combinado das correntes do primário e secundário e o fluxo disperso de primário 


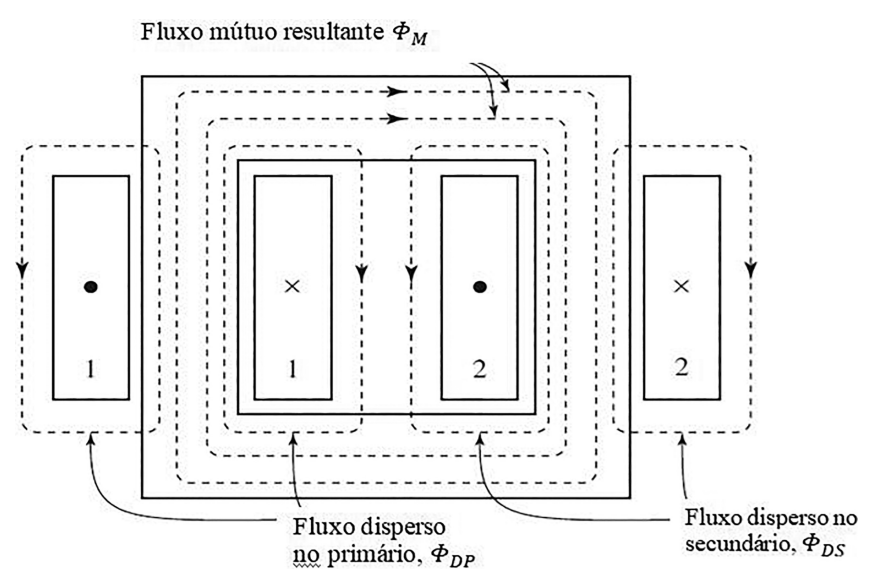

Figura 3: Vista esquemática das linhas de campo magnético (linhas tracejadas) em um transformador. As bobinas são representadas pelos retangulos menores. Os sinais " $x$ " $e$ "•" indicam o sentido da corrente nas bobinas. Adaptado de Stephen D. Umans, 2014 [4].

$\left(\Phi_{D P}\right)$ e secundário $\left(\Phi_{D S}\right)$, que atravessa uma das bobinas, mas não a outra. Este fluxo está relacionado as linhas de campo magnético que deixam o núcleo de ferro, e passam através do ar. Tendo em vista que maior parte das linhas de campo de $\Phi_{D P}$ e $\Phi_{D S}$ estão no ar, estes fluxos serão proporcionais a corrente em seus respectivos enrolamentos. Desta forma, as indutâncias de dispersão nas bobinas do primário $\left(L_{P}\right)$ e secundário $\left(L_{S}\right)$ podem ser definidas como:

$$
L_{P}=\frac{\Phi_{D P}}{i_{P}} \quad \text { e } \quad L_{S}=\frac{\Phi_{D S}}{i_{S}} .
$$

Com isto, as respectivas reatâncias de dispersão, $X_{P}$ e $X_{S}$, são dadas por:

$$
X_{P}=2 \pi f L_{P} \quad \text { e } \quad X_{S}=2 \pi f L_{S} .
$$

No modelo de transformador real, $X_{P}$ se somará em série à resistência ôhmica da bobina do circuito primário $\left(R_{P}\right)$. O mesmo acontecendo no circuito do secundário com relação a $X_{S}$ e $R_{S}$. Na Figura 4 estão apresentadas parte do circuito do primário, com estas componentes. Está representado também a tensão $\varepsilon_{P}$, induzida na bobina do primário, devido à variação do fluxo mútuo resultante que atravessa as bobinas do primário e secundário.

O acento circunflexo em $\hat{V}_{P}$ e $\hat{i}_{P}$ é uma notação comumente utilizada para tratar estas grandezas em sua forma fasorial (veja o Apêndice I). A descrição fasorial simplifica a análise de circuitos contendo impedâncias capacitivas e indutivas [8]. Nas próximas seções utilizase esta notação para a descrição de tensões e correntes.

\subsubsection{Perdas no núcleo e o circuito equivalente}

O fluxo mútuo resultante no núcleo é devido as combinações das $f m m$ 's no primário $\left(N_{P} \hat{i}_{P}\right)$ e secundário

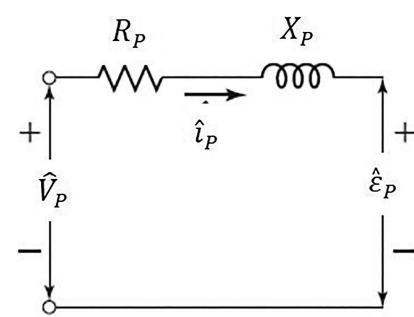

Figura 4: Parte do circuito primário que modela um transformador real. Adaptado de Stephen D. Umans, 2014 [4].

$\left(N_{S} \hat{i}_{S}\right)$. A corrente $\hat{i}_{P}$ no primário deve ser suficiente para magnetizar o núcleo, produzindo um fluxo mútuo resultante, e contrabalancear o efeito da $\mathrm{fmm}$ do secundário, que atua no sentido de desmagnetizar o núcleo. Desta forma, escreve-se a corrente no primário em duas componentes: (i) uma corrente de excitação $\left(\hat{i}_{\Phi}\right)$ necessária para produzir o fluxo mútuo resultante, e (ii) uma componente de carga $\left(\hat{i}_{S}^{\prime}\right)$ que contrabalanceia a corrente, $\hat{i}_{S}$, no secundário. A $\mathrm{fmm}$ resultante no núcleo, advinda da diferença entre $N_{P} \hat{i}_{P}$ e $N_{S} \hat{i}_{S}$, deve ser igual a $f m m$ produzida por $i_{\Phi}$ no primário, tendo em vista que esta corrente é a que produz fluxo no núcleo. Desta forma:

$$
N_{P} \hat{i}_{P}-N_{S} \hat{i}_{S}=N_{P} \hat{i}_{\Phi}
$$

Como $\hat{i}_{P}=\hat{i}_{S}^{\prime}+\hat{i}_{\Phi}$ tem-se na Equação 12 que:

$$
N_{P}\left(\hat{i}_{S}^{\prime}+\hat{i}_{\Phi}\right)-N_{S} \hat{i}_{S}=N_{P} \hat{i}_{\Phi}
$$

Resolvendo para $\hat{i}_{S}^{\prime}$ obtém-se:

$$
\hat{i}_{S}^{\prime}=\frac{N_{S}}{N_{P}} \hat{i}_{S}
$$

Nota-se assim que $\hat{i}_{S}^{\prime}$ é igual a $\hat{i}_{S}$ referida ao primário, como no caso de um transformador ideal.

No núcleo do transformador existem as componentes de perdas por histerese magnética e correntes de Foucault. No modelo que descreve o circuito equivalente do transformador real, estas perdas aparecem como uma resistência de magnetização, $R_{C}$. Há também as perdas devido a reatância de magnetização do núcleo, $X_{m}$, quando uma componente da corrente $\hat{i}_{\Phi}$, é utilizada para magnetizá-lo. O núcleo é então designado por um ramo de excitação no qual parte de $\hat{i}_{\Phi}$, denominada de $\hat{i}_{C}$, atravessa o ramo contendo $R_{C}$, e outra parte, denominada de $\hat{i}_{m}$, atravessa $X_{m}$. O termo $X_{m}$ é dado por:

$$
X_{m}=2 \pi f L_{m}
$$

no qual $L_{m}$ é a indutância de magnetização do núcleo. Assim, no modelo do circuito equivalente, as grandezas $R_{C}$ e $X_{m}$, são associadas em paralelo no ramo de excitação, conforme ilustrado na Figura 5. Como se observa nesta Figura, estes termos estão em paralelo 


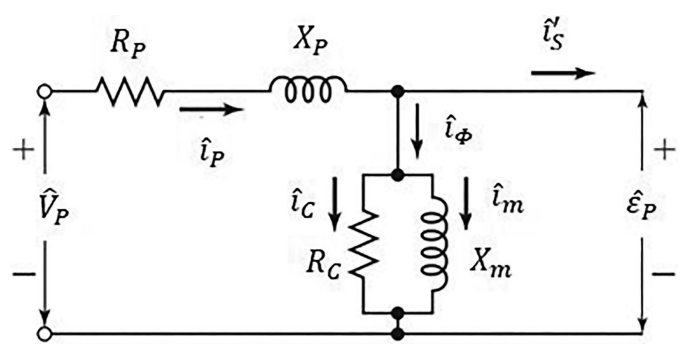

Figura 5: Circuito primário de um transformador real. Adaptado de Stephen D. Umans, 2014 [4].

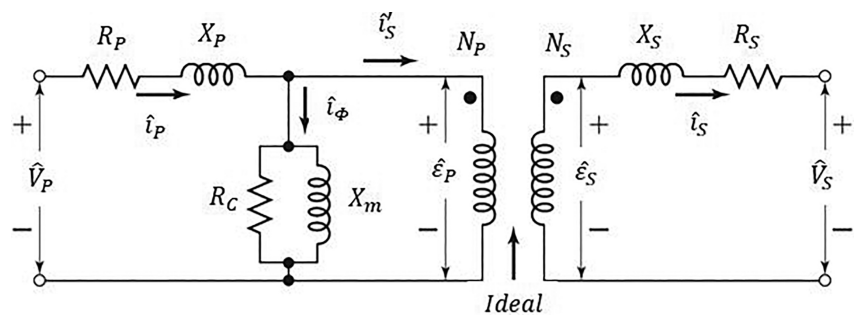

Figura 6: Representação do circuito primário e secundário de um transformador real. Adaptado de Stephen D. Umans, 2014 [4].

com $\varepsilon_{P}$. A combinação em paralelo de $R_{C}$ e $X_{m}$ é denominada de impedância de magnetização, $Z_{\Phi}$.

$\mathrm{Na}$ Figura 6, está representado o circuito equivalente do transformador com todos os componentes do primário e secundário. O fluxo mútuo resultante, $\hat{\Phi}_{M}$, que atravessa ambas as espiras do primário e secundário é o responsável pela indução da tensão em ambas as bobinas, $\varepsilon_{P}$ e $\varepsilon_{S}$. Devido a isso, constata-se que:

$$
\frac{\varepsilon_{P}}{\varepsilon_{S}}=\frac{N_{P}}{N_{S}} .
$$

A Equação 16 juntamente com a Equação 114 são tranformações de tensão e corrente, respectivamente, para o modelo de um transformador ideal. Tem-se assim que o modelo para o transfomador real é equivalente ao de um transformador ideal mais as impedâncias externas.

Para a determinação de impedâncias do transformador e seu rendimento, é usual referenciar as impedâncias à direita ou esquerda do circuito equivalente. Na Figura 7 o circuito equivalente está desenhado com as impedâncias e a tensão do secundário, referenciado ao primário. Portanto, de acordo com o discutido na seção 2.2 no primário estas grandezas são escritas como:

$$
\begin{gathered}
X^{\prime}{ }_{S}=\left(\frac{N_{P}}{N_{S}}\right)^{2} X_{S} \\
R_{S}^{\prime}=\left(\frac{N_{P}}{N_{S}}\right)^{2} R_{S} \\
V^{\prime}{ }_{S}=\frac{N_{P}}{N_{S}} V_{S}
\end{gathered}
$$

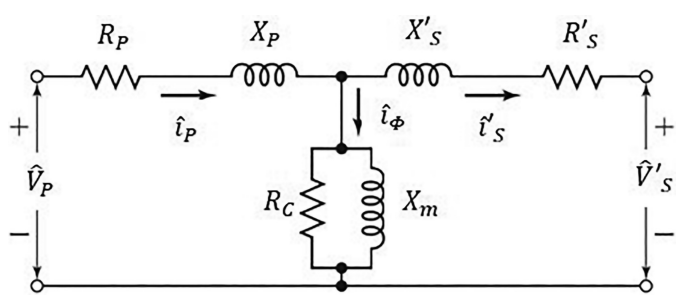

Figura 7: Circuito de um tranformador real com parâmetros do secundário referenciados ao primário. Adaptado de Stephen D. Umans, 2014 [4].

\section{Determinação de Impedâncias}

As determinações das impedâncias do transformador podem ser obtidas por dois tipos de ensaios: curto-circuito e circuito aberto. No primeiro, um curto-circuito é feito em um dos terminais do transformador, possibilitando obter com boa aproximação a impedância das bobinas, devido as reatâncias de disperção e a resistências ôhmicas. No segundo ensaio, com um dos terminais em aberto, obtém-se a impedância relativa ao ramo de excitação da associação em paralelo da resistências e reatâncias de magnetização do núcleo.

\subsection{Ensaio em curto-circuito}

Neste tipo de ensaio, por conveniência, faz-se um curtocircuito no lado de baixa tensão do transformador e alimenta-se o lado de alta tensão, definido neste caso como o primário. A tensão é elevada até que um valor de corrente, igual ao valor nominal de operação, é medida no secundário. Na Figura 8 está representado o circuito equivalente com a impedância do secundário do transformador referida ao lado do primário e um curto-circuito aplicado ao lado do secundário. O curtocircuito no primário é devido a "reflexão" do curtocircuito feito no secundário. Como a impedância $Z_{\Phi}$ do ramo de excitação é muito maior que a impedância devido a reatância de dispersão e a resistência ôhmica do secundário, tem-se que a impedância de curto-circuito $\left(Z_{C C}\right)$ olhando para o primário é, com boa aproximação:

$$
Z_{C C}=R_{P}+j X_{P}+R_{S}^{\prime}+j X_{S}^{\prime}=R_{e q}+j X_{e q} .
$$

Em circuitos elétricos a impedância é um número complexo (veja o Apêndice I). As resistências compõem a parte real da impedância, enquanto as reatâncias são acompanhadas da unidade complexa $j=\sqrt{-1}$.

Nos ensaios de curto-circuito medem-se os valores eficazes de tensão aplicada $\left(V_{C C}\right)$, da corrente de curto circuito $\left(i_{C C}\right)$ e da potência ativa $\left(P_{C C}\right)$. Esta última é medida com um wattímetro, que fornecem também a corrente, tensão e fator de potência (veja a seção 5.2 . Com estas três medições os valores de $Z_{C C}, R_{e q}=$ $R_{C C}$ e $X_{e q}=X_{C C}$, referenciados ao primário, são 


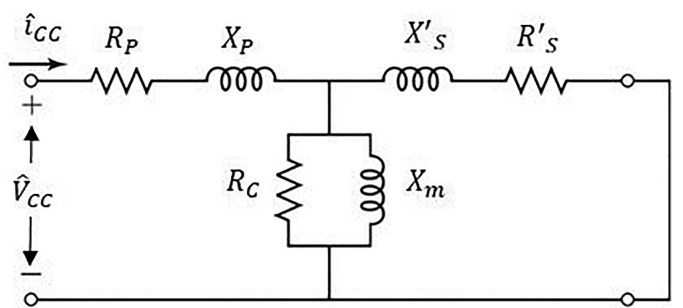

Figura 8: Circuito equivalente com a impedância do secundário do transformador referida ao lado do primário e um curto circuito no secundário. Adaptado de Stephen D. Umans, 2014 [4].

determinados por (veja o Apêndice II):

$$
\begin{gathered}
\left|Z_{C C}\right|=\frac{V_{C C}}{i_{C C}}, \\
R_{C C}=\frac{P_{C C}}{i_{c c}^{2}}, \\
X_{C C}=\sqrt{\left|Z_{C C}\right|^{2}-R_{C C}^{2}} .
\end{gathered}
$$

\subsection{Ensaio em circuito aberto}

Neste ensaio os terminais do lado de alta tensão são mantidos abertos enquanto alimenta-se o lado de baixa tensão, que neste caso é definido como o primário. Como não há nenhuma corrente no secundário, obtém-se o valor eficaz de $\hat{i}_{\Phi}$ no circuito primário. Normalmente a tensão nominal é escolhida, de modo a assegurar que o núcleo, e por consequência a reatância de magnetização, opere em condições de fluxo próximo ao utilizado em carga de operação. A tensão nominal é aquele que o transformador foi projetado para trabalhar. Nela, leva-se em consideração a corrrente máxima que o enrolamento suporta bem como a tensão de isolamento do material isolante.

Na Figura 9 está representado o circuito equivalente com a impedância do secundário referida ao lado do primário e o secundário em aberto. Como a impedância no ramo de excitação é muito maior que a impedância da bobina de primário (devido a $R_{P}$ e $X_{P}$ ), temse que queda de tensão na bobina é desprezível em relação ao ramo de excitação. Portanto a tensão $V_{C A}$ do primário é praticamente igual a $f e m, \varepsilon_{C A}$, no ramo de excitação. Nessas condições, a impedância $\left(Z_{C A}\right)$ no ensaio de circuito aberto é dada, com boa aproximação, pela equação:

$$
Z_{C A}=\frac{R_{C} \cdot j X_{m}}{R_{C}+j X_{m}}
$$

Nesta Equação foi utilizada a natureza complexa da impedância reativa (veja o Apêndice I.

A instrumentação utilizada no ensaio de circuito aberto, é semelhante ao de curto-circuito. Medem-se os valores eficazes de tensão aplicada $\left(V_{C A}\right)$, da corrente de circuito aberto $\left(i_{C A}\right)$ e da potência ativa $\left(P_{C A}\right)$.

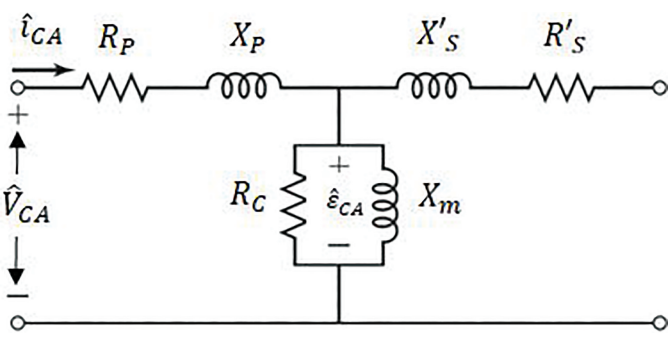

Figura 9: Circuito equivalente com a impedância do secundário referida ao lado do primário e o secundário em aberto. Adaptado de Stephen D. Umans, 2014 [4].

Com estas três medições os valores de $\left|Z_{C A}\right|, R_{C}$ e $X_{m}$, referenciados ao primário, são dadas por (veja o Apêndice II):

$$
\begin{gathered}
\left|Z_{C A}\right|=\frac{V_{C A}}{i_{C A}}, \\
R_{C}=\frac{V_{C A}{ }^{2}}{P_{C A}}, \\
X_{m}=\frac{1}{\sqrt{\left(1 /\left|Z_{C A}\right|\right)^{2}-\left(1 / R_{C}\right)^{2}}} .
\end{gathered}
$$

O ensaio de circuito aberto pode ser utilizado para a obtenção das perdas no núcleo, em cálculos de rendimento e para a verificação do módulo da corrente de excitação (veja a seção 5.3 .

\section{Procedimentos Experimentais para a Confecção do Transformador}

A construção do transformador (Figura 10(a)-(h)), se deu em três etapas: os carreteis, as bobinas do primário e secundário e o núcleo. Para a construção do núcleo, foram utilizadas chapas finas de aço-Si que estavam sucateada em uma oficina de enrolamento de motores e transformadores, localizada no município de São Mateus, ES. Estas placas também foram utilizadas para a construção do carretel das bobinas. Na Figura 10(a) é mostrado um conjunto de chapas finas, que formam um bloco com dimensões de 3,0 × 3,0 × 15,0 cm. Este bloco foi utilizado apenas em uma etapa da construção dos carreteis. Suas dimensões de largura e espessura definem a área de seção reta do núcleo.

Para o enrolamento das bobinas do primário e secundário, foi utilizado fio de cobre esmaltado, com numeração AWG 21. Os enrolamentos foram feitos manualmente, conforme ilustrado na Figura 11(a). A bobina do primário foi definida com 350 espiras e para o secundário foi utilizada uma bobina com ramificações contendo 90, 150, 225, 300 e 450 espiras. Com isto pode-se analizar como as tensões são transformadas com relação ao número de espiras, em um experimento simples com o secundário aberto. Após o enrolamento de uma das camadas (Figura 11(b)), uma folha de papel A4 

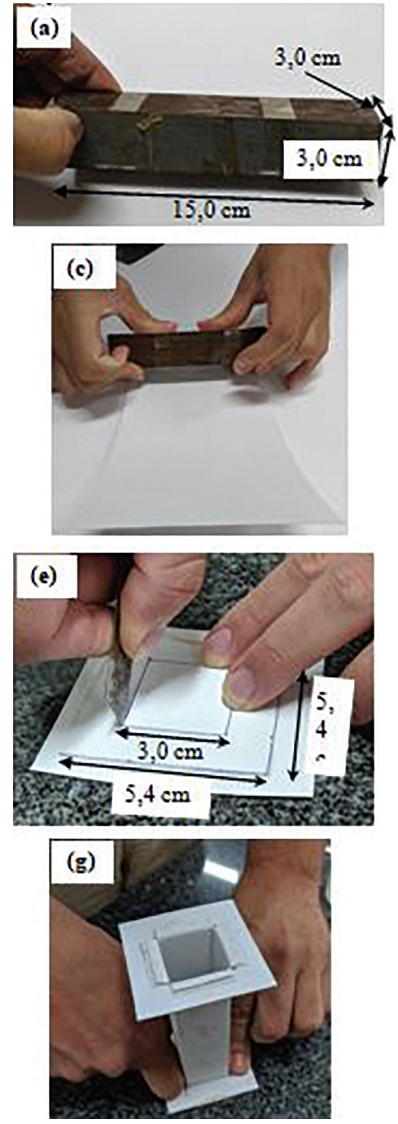

Figura 10: (a) Bloco utilizado para construção do carretel. (b)(d) Etapas da construção do carretel. (e)-(f) Contrução da aba do carretel. (g)-(h) O carretel construído.
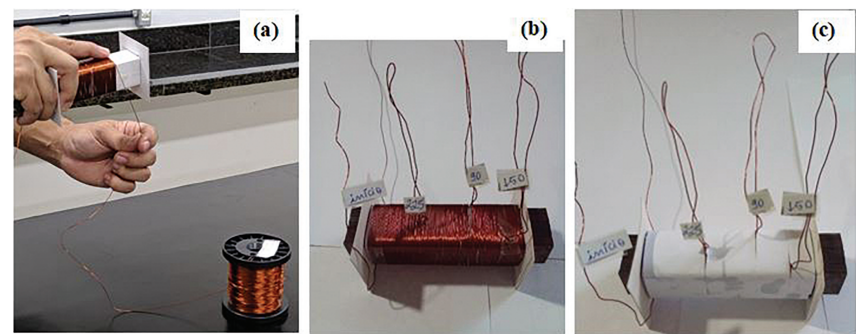

Figura 11: (a) Enrolamento manual das bobinas. (b)-(c) Etapas da construção do secundário com diversas espiras.

foi utilizada para melhor fixação das espiras e facilitar o enrolamento de outra camada de espiras (Figura 11.(c)).

$\mathrm{Na}$ Figura 12 são mostradas as chapas maiores e menores utilizadas para a confecção do núcleo tipo "U" e suas dimensões. Estas foram agrupadas em conjuntos de 10 unidades e para isto foi utilizado fita adesiva transparente.

A montagem do transformador foi realizada conforme as imagens da Figura 13(a)-(f). Inicialmente, com as chapas, foram feitos retângulos do tipo $\mathrm{R}_{1}$ (Figura 13(a)) e $R_{2}$ (Figura 13(b)) e estes foram empilhados um sobre o outro, de forma alternada até se obter a espessura

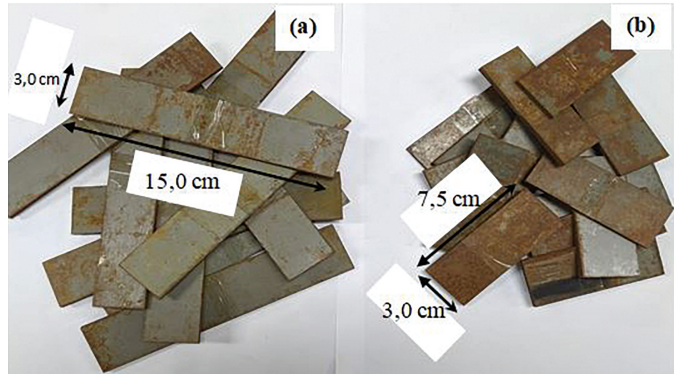

Figura 12: (a) Chapas maiores e (b) menores utilizadas na confecção do núcleo.
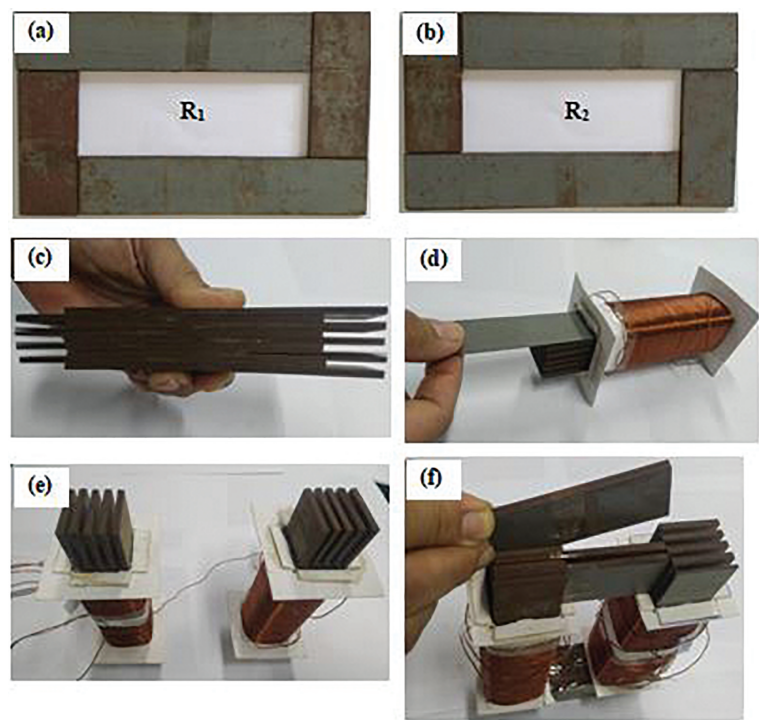

Figura 13: Configuração de camadas dos tipos (a) e (b) sobrepostas na construção do núcleo. (c) Forma do comprimento maior do núcleo. (d) Inserção de lâminas para cobrir espaços remanescentes no núcleo. (e) As bobinas com os núcleos. (f) Etapa final da construção do transformador.

desejada para o núcleo. Com esta construção as frestas entre um conjunto de chapas e outro foram cobertas. Após o empilhamento, as lâminas menores à esquerda e a direita foram retiradas para a insersão nas bobinas do primário e secundário. O resultado que se obtém está mostrado na Figura 13(c). Este conjunto de chapas deve ser inserido em uma das bobinas conforme a Figura 13 (d) e se ficar algum espaço, deve-se inserir lâminas ate que fique bem ajustado. Na Figura 13. (e) pode-se observar as bobinas do primário e secundário após a insersão dos respectivos núcleos. Na ultima etapa, basta inserir as chapas menores, nos espaços entre uma chapa e outra, completando o núcleo em U e o transformador (veja a Figura $13(\mathrm{f})$ ).

\section{Resultados Experimentais}

As práticas experimentais que podem ser realizadas com o transformador aqui construído são: (i) com as bobinas 

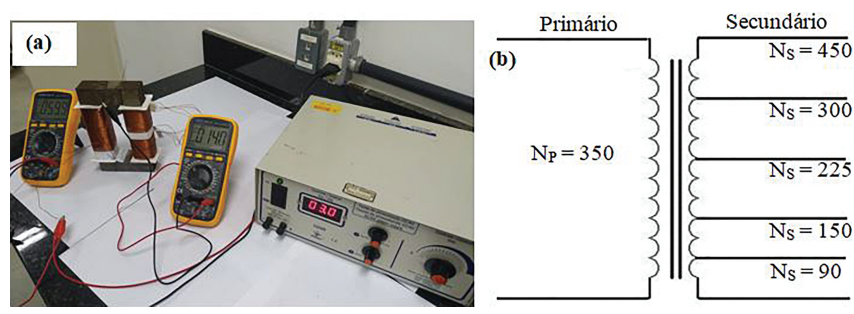

Figura 14: (a) Montagem experimental para análise da tensão no secundário com o número de espiras. (b) Ilustração esquemática do primário e secundário e seus números de espiras.

do secundário em aberto, a verificação da relação entre a tensão no primário e secundário e os respectivos número de espiras em suas bobinas, (ii) determinação, no ensaio de curto circuito, da impedância equivalente das resistências das bobinas do primário e secundário $\left(R_{e q}\right)$ e de suas reatâncias de dispersão equivalente $\left(X_{e q}\right)$, (iii) determinação, no ensaio de circuito aberto, da resistência de magnetização no núcleo $\left(R_{C}\right)$ e da reatância de magnetização $\left(X_{m}\right)$ e, por fim, (iv) obtenção do rendimento.

\subsection{A relação de transformação da tensão com o número de espiras}

Na Figura 14(a) está apresentado o aparato experimental utilizado para a verificação da transformação da tensão com o número de espiras no secundário. Um variac foi utilizado para alimentar a bobina do primário, em um valor definido de tensão, que medido com um multímetro marca $V_{P} \approx 60,0 \mathrm{~V}$. Com um segundo multímetro, mediu-se os valores de tensão para cada uma das bobinas do secundário $V_{S}$, representadas na ilustração do transformador da Figura 14(b).

No gráfico da Figura 15 está representado a relação entre $V_{S} / V_{P}$ e $N_{S} / N_{P}$. A relação entre estas grandezas é diretamente proporcional, visto o comportamento linear entre elas. $\mathrm{O}$ coeficiente angular da reta, $\alpha \approx 0,9$, está muito próximo ao que se obteria em um transformador ideal, $\alpha=1$. A pequena diferença entre estes valores decorre do fato de que nem todo o fluxo magnético induzido no primário é conduzido pelo núcleo ao enrolamento do secundário devido a dispersão e, também devido as correntes parasitas induzidas no núcleo. Assim, a tensão induzida no secundário não acontece em sua totalidade como em um transformador ideal.

\subsection{O transformador em carga}

Para se verificar um caso particular das potências ativas no primário e secundário do transformador e seu rendimento, a bobina do secundário com $N_{S}=225$ espiras foi conectada a uma carga puramente resistiva de $44 \Omega$ (dois resistores de porcelana $22 \Omega / 15 \mathrm{~W}$ em série). A tensão aplicada ao primário foi de $V_{P} \approx 56,0 \mathrm{~V}$ e observouse que no secundário esta foi de $V_{P} \approx 29,1 \mathrm{~V}$. Para

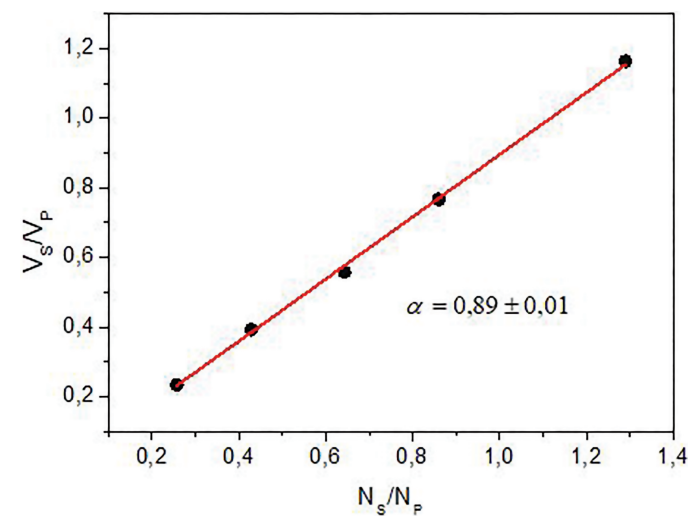

Figura 15: Razão entre as tensões no primário e secundário em função da razão entre seus respectivos números de espiras. A letra " $\alpha$ " representa a inclinação da reta.

uma tensão superior a este valor, foi observado um leve aquecimento das bobinas, acompanhado de vibrações das chapas do núcleo. Desta forma, para valores de $V_{P}>56,0 \mathrm{~V}$ uma quantidade considerável de energia é dissipada em calor e vibração. Definiu-se assim que, para o caso em particular com $N_{S}=225$ espiras, e carga resistiva de $44 \Omega$, o transformador opera com tensão nominal de carga no secundário de $V_{S} \approx 29,1 \mathrm{~V}$.

$\mathrm{Na}$ Figura 16 está apresentado o ensaio com o transformador em carga e na Figura 17um desenho esquemático utilizando simbologias. O variac foi ligado em paralelo à bobina do primário e um dos terminais da bobina do secundário, com $N_{S}=225$ voltas, foi enrolado 11 vezes ao alicate do wattímetro, a fim de se amplificar e realizar a leitura da corrente elétrica fornecida por este aparelho. Isto foi necessário devido ao fato de o wattímetro disponível ser do tipo alta potência e com precisão de escala de $0,01 \mathrm{~kW}$. Após leitura, o valor de corrente deve ser dividido por 11. A baixa precisão do wattímetro, relativa ao nosso experimento, impossibilitou a obtenção de parâmetros elétricos com uma precisão mais adequada. Entretanto, foi suficiente para uma boa estimativa de potências e rendimentos $(\eta)$ do transformador com $N_{S}=225$ espiras. Os terminais de tensão do wattímetro no primário são conectados em paralelo com a bobina do primário (veja a Figura 17). Para a leitura das grandezas elétricas no secundário do transformador, utilizando o wattímetro, um dos terminais foi enrolado 11 vezes no alicate do wattímetro. Este mesmo terminal, após passar pelo wattímetro, foi utilizado juntamente com o outro terminal da mesma bobina para alimentar o resistor de $22 \Omega$. Os terminais de tensão do wattímetro são conectados em paralelo com o resistor.

Na Tabela 1 estão apresentadas as leituras obtidas com o wattímetro para os lados do primário e secundário do transformador. Os parâmetros elétricos obtidos foram: tensão $(V)$, corrente $(i)$, potência ativa $\left(P_{A t i}\right)$, potência aparente $\left(P_{A p a}\right)$ e Fator de Potência $(F P)$. O valor da corrente no secundário $\approx 0,68 \mathrm{~A}$, foi definida 


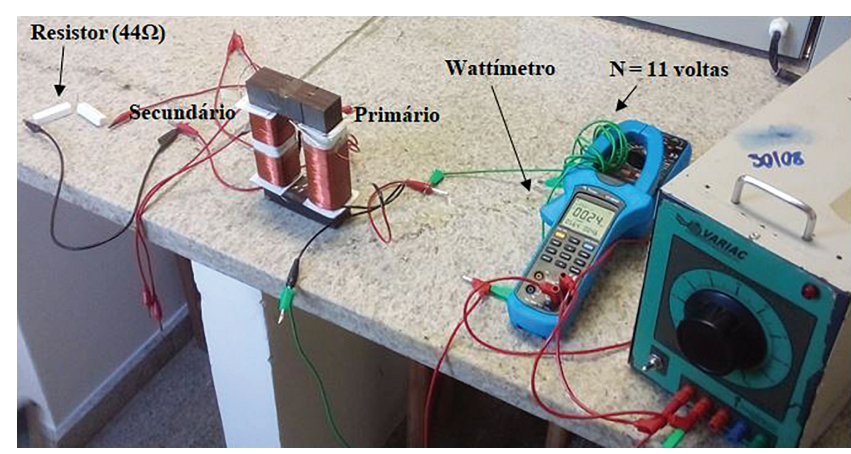

Figura 16: Montagem experimental para a obtenção de parâmetros do transformador em carga.

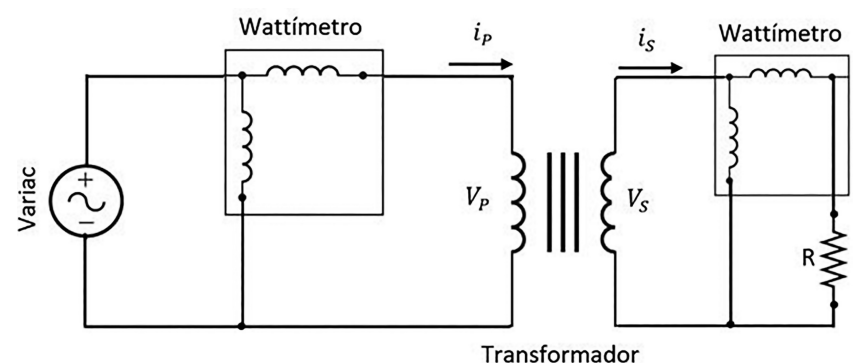

Figura 17: O circuito para o estudo do transformador com carga. O wattímetro é representado por um quadrado contendo dois enrolamentos. Para o caso do primário, o enrolamento em paralelo com esta bobina mede a tensão $V_{P}$ e o enrolamento em série, a corrente $i_{P}$. Fonte: $o$ autor.

Tabela 1: Parâmetros elétricos obtidos com o primário alimentado em $V_{P} \approx 60,0 \mathrm{~V}$ e uma carga resistiva de em $R \approx 44 \Omega$.

\begin{tabular}{lccccc}
\hline $\begin{array}{c}\text { Medidas no: } \\
\downarrow\end{array}$ & $V(\mathrm{~V})$ & $i(\mathrm{~A})$ & $P_{\text {Ati }}(\mathrm{W})$ & $P_{\text {Apa }}(\mathrm{W})$ & $F P$ \\
\hline Primário & 55,9 & 0,44 & 21,9 & 24,6 & 0,92 \\
\hline Secundário & 29,4 & 0,68 & 20,0 & 20,0 & 0,99 \\
\hline
\end{tabular}

como a corrente nominal, supondo que o transformador opere tensão nominal $\approx 56,0 \mathrm{~V}$.

De acordo com a Tabela 1] obtém-se: $V_{P} / V_{S} \approx$ 1,9 e $N_{P} / N_{S} \approx 1,6$. O fato de estas relações não formarem uma igualdade, que seria verdade para um transformador ideal, indicam a existência de queda de tensão nas bobinas, devido a impedância existente nela e no núcleo (veja a seção 3). Por outro lado a relação: $i_{P} / i_{S} \approx 0,65$ e $N_{S} / N_{P} \approx 0,64$ (lembrando que estas razões são inversamente proporcionais) formam uma igualdade, como no caso de um transformador ideal, o que indica uma corrente de excitação $i_{\Phi} \approx 0$. Os fatores de potência obtido pelo wattímetro (Tabela 1), estão de acordo com os valores teóricos dados por: $F P=P_{A t i} / P_{A p a}$ (veja o Apêndice II). Para o primário $F P=21,9 / 24,6 \approx 0,90$ e para o secundário $F P \approx 0,99$. No circuito do secundário, mede-se a corrente e a tensão em uma carga puramente resistiva, então o ângulo entre tensão e corrente é nulo e, portanto, $F P \approx 1$. Já no primário, mede-se a corrente e a tensão em um circuito constituído pelas resistências ôhmicas do primário e reatâncias indutivas, devido aos fluxos de dispersão e impedâncias do núcleo. Logo, espera-se que $F P<1$ (carga indutiva). O rendimento $(\eta)$ do transformador, nessas condições, é calculado como [9]:

$$
\eta=\frac{P_{A t i} \text { Secundário }}{P_{A t i} \text { Primário }}
$$

e assim, utilizando as potências na Tabela 1 têm-se que $\eta \approx 90 \%$. Este valor está na ordem de grandezas para transformadores comerciais 8).

\subsection{Ensaios de curto circuito e circuito aberto}

Na Tabela 2 estão apresentados os resultados experimentais obtidos dos ensaios em Curto Circuito (CC) e Circuito Aberto (CA). Os respectivos ensaios foram realizados seguindo as discussões apresentadas nas seções 3.1 e 3.2 Para a medição das correntes foram utilizados $N=12$ voltas no alicate do wattímetro. No ensaio de CC o valor de corrente utilizado foi $\approx 0,67 \mathrm{~A}$, praticamente o mesmo valor, definido como o nominal com o transformador em carga. De forma semelhante, no ensaio de CA o valor de tensão escolhido foi $\approx 29,1 \mathrm{~V}$, o mesmo definido como tensão nominal com o transformador em carga. A escolha de valores nominais seguem os protocolos de ensaios técnicos de transformadores, permitindo obter de forma coerente seus rendimentos quando estes operam com seus valores nominais de tensão e corrente [3, 8].

Com base nos valores apresentados na Tabela 2 utilizando as Equações (21), 22) e (23) para o ensaio de curto circuito, obtém-se os valores para $Z_{C C} \approx 21 \Omega$, $R_{C C} \approx 6 \Omega$ e $X_{C C} \approx 20 \Omega$, respectivamente. Adicionalmente utilizando as Equações 25,26 e 27 para o ensaio de circuito aberto, obtém-se, respectivamente $\left|Z_{C A}\right| \approx 170 \Omega, R_{C} \approx 508 \Omega$ e $X_{m} \approx 180 \Omega$. Observa-se uma maior ordem de grandeza dos parâmetros associados ao ensaio de circuito aberto (parâmetros do núcleo do transformador), o que justifica a aproximação de que a queda de tensão, devido a impedância das bobinas, é desprezível em relação a impedância do núcleo.

Com as grandezas elétricas obtidas na Tabela 2 , podese também estimar de outra forma o rendimento do

Tabela 2: Parâmetros obtidos dos ensaios de curto circuito e circuito aberto.

\begin{tabular}{lccc}
\hline \multicolumn{4}{c}{ Curto Circuito } \\
\hline$V_{C C}(\mathrm{~V})$ & $i_{C C}(\mathrm{~A})$ & $P_{C C}{ }^{A t i}(\mathrm{~W})$ & $P_{C C}{ }^{A p a}(\mathrm{~W})$ \\
\hline \multicolumn{5}{c}{0,67} & 2,5 & 9,5 \\
\hline \multicolumn{4}{c}{ Circuito Aberto } \\
\hline$V_{C A}(\mathrm{~V})$ & $i_{C A}(\mathrm{~A})$ & $P_{C A}{ }^{A t i}(\mathrm{~W})$ & $P_{C A}{ }^{A p a}(\mathrm{~W})$ \\
\hline 29,1 & 0,17 & 1,7 & 5,0 \\
\hline
\end{tabular}


transformador, com a Equação 8]:

$$
\eta=\frac{P_{A t i}{ }^{\text {Secundário }}}{P_{A t i}{ }^{\text {Secundário }}+P_{C C}{ }^{A t i}+P_{C A}{ }^{A t i}},
$$

no qual o termo do denominador nessa equação representa a potência ativa no primário. O valor a ser utilizado para $P_{A t i}$ Secundário deve ser o mesmo do valor nominal, e assim, do ensaio com o transformador em carga, 20 W (veja a Tabela 1). O rendimento calculado foi de $\eta \approx 83 \%$ um valor ligeiramente inferior ao do calculado utilizando-se a Equação (28). Esta diferença é devido a imprecisão do wattímetro utilizando, para a medição dos parâmetros elétricos do transformador. Portanto, o rendimento está compreendido na faixa de valores entre $80 \%-90 \%$.

\section{Considerações Finais}

Através de um experimento simples, foi possível obter grandezas físicas pertinentes à transformação de tensão, potência e rendimento de um transformador. Foi necessária uma inclusão teórica sobre o funcionamento de um transformador real, que geralmente não é abordada em livros textos de física básica para estudantes de graduação. A teoria que aqui foi apresentada ajuda os estudantes a compreenderem porque a transformações de tensão, não ocorrem exatamente como em um transformador ideal. Grandezas físicas presentes como as reatâncias de dispersão nas bobinas e as impedâncias do núcleo devem ser levadas em consideração no cálculo das transformações de tensão e obtenção do rendimento. Para o caso particular do transformador aqui construído e com o secundário em 225 espiras, os denominados ensaios de curto circuito e circuito aberto possibilitaram obter as respectivas impedâncias equivalentes das bobinas e do núcleo do transformador. Conclui-se que este trabalho é de grande importância para o ensino prático de eletromagnetismo, levando em consideração que o transformador é um equipamento de extrema importância na transmissão da energia elétrica, presente no cotidiano de uma população.

\section{Agradecimentos}

A equipe de trabalho agradece aos programas PIIC de Pesquisa e Pós-graduação da UFES e PROGRAD/UFES pela concessão de bolsas de estudos e financiamento desta pesquisa. Agradecemos ao IFES de São Mateus - ES pelo uso de instrumentos laboratoriais.

\section{Material suplementar}

O seguinte material suplementar está disponível online:

\section{Apêndice I. Notação Fasorial para Análise de Circuitos}

Uma função senoidal de uma fonte de tensão, em regime permanente, pode ser escrita em termos de seno ou cosseno. Escolhendo-se a forma em cosseno, tem-se:

$$
V=V_{m} \cos \left(\omega t+\theta_{v}\right)
$$

nos quais $V_{m}$ é a amplitude da tensão, $\omega$ é a frequência angular e $\theta_{v}$ o ângulo de fase. Esta equação é igual a parte real (designado por: $\mathcal{R}$ ) da identidade de Euler que relaciona uma função exponencial complexa ( $\mathrm{com} j=$ $\sqrt{-1}$ ) com uma função trigonométrica:

$$
V=V_{m} \cos \left(\omega t+\theta_{v}\right)=\mathcal{R}\left[V_{m} e^{j\left(\omega t+\theta_{v}\right)}\right],
$$

que pode ser reescrita como:

$$
V=\mathcal{R}\left[V_{m} e^{j \theta_{v}} e^{j \omega t}\right] .
$$

Nesta equação, a quantidade complexa:

$$
\hat{V}=V_{m} e^{j \theta_{v}},
$$

que contém informações sobre a amplitude e ângulo de fase, é por definição a representação fasorial ou a transformada fasorial $(\mathcal{P})$ da função senoidal I.1

$$
\hat{V}=\mathcal{P}\left[V_{m} \cos \left(\omega t+\theta_{v}\right)\right]=V_{m} e^{j \theta_{v}} .
$$

A transformada fasorial $\mathcal{P}\left[V_{m} \cos \left(\omega t+\theta_{v}\right)\right]$ transfere a função senoidal do domínio do tempo para o domínio dos números complexos, também chamado de domínio das frequências. A notação $\hat{V}=V_{m} e^{j \theta_{v}}$ pode também ser escrita na forma retangular como:

$$
\hat{V}=V_{m}\left(\cos \theta_{v}+j \operatorname{sen} \theta_{v}\right) .
$$

Para passar do domínio das frequências para o domínio do tempo, aplica-se a transformada fasorial inversa (designada por $\mathcal{P}^{-1}$ ) em $\hat{V}=V_{m} e^{j \theta_{v}}$ :

$$
\mathcal{P}^{-1}\left(V_{m} e^{j \theta_{v}}\right)=\mathcal{R}\left[V_{m} e^{j \theta_{v}} e^{j \omega t}\right]=V_{m} \cos \left(\omega t+\theta_{v}\right) .
$$

A vantagem de se trabalhar com a notação fasorial é que se reduz os cálculos trabalhosos com funções senoidais em circuitos, à álgebra dos números complexos [8].

Demonstra-se que no domínio dos números complexos as relações entre os fasores de tensão, de corrente e de grandezas que caracterizam os elementos de um circuito são escritas como:

$$
\hat{V}=R \hat{i},
$$

(elemento resistivo)

$$
\hat{V}=j \omega L \hat{i},
$$

(elemento indutivo)

$$
\hat{V}=-j \frac{1}{\omega C} \hat{i},
$$

(elemento capacitivo) 
nos quais $R$ é a resistência de um elemento resistivo e os termos complexos $j X_{L}=j \omega L \mathrm{e}-j X_{C}=-j 1 / \omega C$ representam as impedâncias indutivas e capacitivas respectivamente. Demonstra-se que utilizando a notação fasorial em um circuito contendo resistores, indutores e capacitores com associações em série, paralelo ou mista, os cálculos para se obter as impedâncias equivalentes seguem a mesma regra utilizada em circuitos que contém apenas elementos resistivos [8]. Após operações utilizando números complexos para resolver circuitos contendo os elementos supracitados, basta aplicar a transformada inversa $\left(\mathcal{P}^{-1}\right)$ nas partes dos circuitos para a obtenção de informações no domínio dos tempos. De uma forma geral, uma impedância é escrita como:

$$
Z=R+j X
$$

No qual a parte real é representada por resistências equivalentes, e a imaginária por reatâncias equivalentes.

\section{Apêndice II. Potência em CA e o Triângulo das Potências}

A potência em qualquer instante de tempo em um circuito CA é dada por:

$$
P=V \cdot i
$$

nos quais:

$$
\begin{gathered}
V=V_{m} \cos (\omega t+\theta), \\
i=i_{m} \cos \omega t
\end{gathered}
$$

e $\theta=\theta_{v}-\theta_{i}$ é a diferença entre os ângulos de fase da tensão $\left(\theta_{v}\right)$ e corrente $\left(\theta_{i}\right)$. Nestas equações escolheu-se para a origem da contagem do tempo, o instante em que a corrente passa por um máximo (instante $t=0$ ). Substituindo as Equações III.2) e III.3 em (II.1), obtémse:

$$
P=V_{m} i_{m} \cos (\omega t+\theta) \cos \omega t
$$

Utilizando identidades trigonométricas convenientes a Equação II.4 pode ser escrita como:

$$
\begin{aligned}
P= & \frac{V_{m} i_{m}}{2} \cos \theta+\frac{V_{m} i_{m}}{2} \cos \theta \cos 2 \omega t \\
& -\frac{V_{m} i_{m}}{2} \operatorname{sen} \theta \operatorname{sen} 2 \omega t .
\end{aligned}
$$

A potência média, fornecida a um circuito, durante um período $T$, é calculada como:

$$
P_{m}=\frac{1}{T} \int_{T_{o}}^{T_{o}+T} P d t,
$$

em que utilizando a Equação II.5 obtém-se:

$$
P_{m}=P_{a t i}=\frac{V_{m} i_{m}}{2} \cos \theta \text {. }
$$

Esta potência também é conhecida como potência ativa e representa a potência que é transformada de elétrica para outras formas de energia. Ela está associada ao trabalho útil. Sua unidade de medição é o watt (W). A potência instantânea (Equação (II.5)) é dada então por:

$$
P=P_{m}+P_{m} \cos 2 \omega t-Q \operatorname{sen} 2 \omega t,
$$

no qual a grandeza $Q$ é denominada de potência reativa. Esta grandeza é escrita como:

$$
Q=\frac{V_{m} i_{m}}{2} \operatorname{sen} \theta
$$

Em circuitos puramente resistivos, tem-se que $\theta=0^{\circ} \mathrm{e}$, portanto, $Q=0$. Assim a potência média é a potência ativa dada pela Equação (II.7). Por outro lado, em circuitos puramente capacitivos ou indutivos no qual $|\theta|=90^{\circ}$, tem-se que $P_{a t i}=0$ e apenas a potência reativa oscila no tempo com frequência $2 \omega$. A média temporal em um ciclo é nula e, portanto, essa potência oscila entre a fonte e os elementos capacitivos ou indutivos, não sendo responsável por uma transformação de energia útil. Para distinguir essa potência da potência ativa, utiliza-se para sua medição a unidade de voltampère reativo (VAR).

A razão entre as Equações (II.9) e III.7 permite uma interpretação geométrica entre as potências de um circuito:

$$
\operatorname{tg} \theta=\frac{Q}{P_{m}}
$$

sendo possível representá-las através do triângulo das potências:

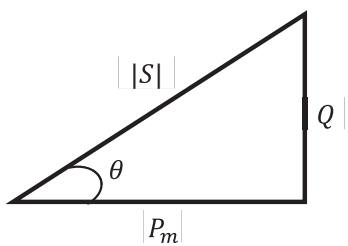

Figura II.1: Triângulo de potências de um circuito CA em regime permanente.

Na Figura II.1 a grandeza $|S|$ é a denominada como o módulo da potência aparente, sendo calculada como:

$$
|S|=P_{a p a}=\sqrt{P_{m}^{2}+Q^{2}},
$$

e a grandeza $\cos \theta=P_{m} /|S|$ é denominada de fator e potência (FP). Tem-se que $\theta>0$ (corrente atrasada em relação a tensão) para circuitos indutivos, e então $\mathrm{FP}>0$. O contrário ocorre para circuitos capacitivos. A potência aparente não tem um significado físico, porém a sua utilização no domínio dos números complexos simplifica a obtenção da potência média, ao trocar equações trigonométricas complicadas por 
simples equações algébricas. Sua unidade de medição é o volt-ampère (VA). A potência aparente, tem a forma complexa dada por:

$$
S=P_{m}+j Q
$$

Em sua forma fasorial, esta potência pode ser calculada de acordo com [8]:

$$
S=\frac{1}{2} \hat{V} \hat{i}^{*}
$$

no qual $\hat{V}=V_{m} e^{j \theta_{v}}$ é a tensão fasorial, e $\hat{i}^{*}=i_{m} e^{-j \theta_{i}}$ é o conjugado complexo da corrente fasorial. Esta última equação pode ser reescrita em temos de valores eficazes:

$$
S=\hat{V}_{e f} \hat{i}_{e f}^{*}
$$

no qual $\hat{V}_{e f}=\frac{V_{m}}{\sqrt{2}} e^{j \theta_{v}}$ e $\hat{i}_{e f}=\frac{i_{m}}{\sqrt{2}} e^{j \theta_{i}}$.

Considerando que:

$$
\hat{V}_{e f}=Z \hat{i}_{e f}
$$

em que $Z=R+j X$ (Equação (I.10), a potência aparente pode assumir a forma:

$$
S=\left|\hat{i}_{e f}\right|^{2} R+j\left|\hat{i}_{e f}\right|^{2} X
$$

no qual $\hat{i}_{e f}=\frac{i_{m}}{\sqrt{2}} e^{j \theta_{i}}$ é a corrente fasorial eficaz e $\left|\hat{i}_{e f}\right|^{2}=$ $\frac{1}{2} i_{m}{ }^{2}$. Outra forma de representar a potência aparente é:

$$
S=\frac{\left|\hat{V}_{e f}\right|^{2}}{Z^{*}},
$$

que assume a forma geral $S=P+j Q$. Se $Z$ é um elemento resistivo puro, então:

$$
S=P=\frac{\left|\hat{V}_{e f}\right|^{2}}{R},
$$

e se $Z$ é um elemento reativo puro, então:

$$
S=j Q=j \frac{\left|\hat{V}_{e f}\right|^{2}}{X} .
$$

No ensaio de curto-circuito (seção 3.1), a Equação (21) pode ser deduzida da Equação (II.15), multiplicando ambos os membros dessa equação por $V_{e f}^{*}=Z^{*} \hat{i}_{e f}^{*}$ :

$$
\hat{V}_{e f} \hat{V}_{e f}^{*}=Z \hat{i}_{e f} Z^{*} \hat{i}_{e f}^{*}
$$

e levando em consideração que $\hat{V}_{e f} \hat{V}_{e f}^{*}=V_{e f}^{2}$, obtém-se:

$$
V_{e f}^{2}=Z^{2} i_{e f}^{2}
$$

Desta forma:

$$
Z_{C C}=\frac{V_{C C}}{i_{C C}}
$$

em que foi feito $V_{e f}=V_{C C}, i_{e f}=i_{C C}$ e $Z=Z_{C C}$. Adicionalmente, a Equação 22 pode ser deduzida diretamente da equação (II.16). Como mede-se a potência ativa, dado pelo primeiro termo dessa Equação, tem-se:

$$
P_{m}=\left|\hat{i}_{e f}\right|^{2} R,
$$

e assim:

$$
R_{C C}=\frac{P_{C C}}{i_{C C}^{2}},
$$

em que foi feito $R=R_{C C}, P_{m}=P_{C C}$ e $\left|\hat{i}_{e f}\right|^{2}=$ $i_{C C}{ }^{2}$. Por fim, a Equação (23) pode ser obtida da Equação (I.10), calculando seu módulo:

$$
|Z|^{2}=|R|^{2}+|X|^{2}
$$

Dessa forma tem-se que:

$$
X_{C C}=\sqrt{\left|Z_{C C}\right|^{2}+\left|R_{C C}\right|^{2}}
$$

em que foi feito $Z=Z_{C C}, X=X_{C C}$ e $R=R_{C C}$.

Continuando com o ensaio de circuito aberto da seção 3.2 a dedução da Equação 25 é feita de forma semelhante à dedução da Equação (21) supracitada. Por outro lado, na dedução da Equação 26, utilizase a Equação (II.18, no qual $P=P_{C A}$ é a potência ativa medida. Por fim, na dedução da Equação 27. multiplica-se primeiro o numerador e o denominador da Equação (24) por: $R-j X_{m}$ :

$$
Z=\frac{R j X_{m}}{R+j X_{m}} \cdot \frac{R-j X_{m}}{R-j X_{m}},
$$

que após algumas álgebras, obtém-se:

$$
Z=\frac{j R^{2} X_{m}+R X_{m}^{2}}{R^{2}+X_{m}{ }^{2}} .
$$

O módulo dessa equação é calculado como $|Z|=$ $\sqrt{Z \cdot Z^{*}}$, o que fornece:

$$
|Z|=\sqrt{\frac{R^{2} X_{m}{ }^{2}\left(R^{2}+X_{m}^{2}\right)}{\left(R^{2}+X_{m}{ }^{2}\right)^{2}}} .
$$

Resolvendo esta equação para $X_{m}$ e simplificando seus termos, chega-se a Equação (27) da seção 3.2

\section{Referências}

[1] R.A. Mion e J.A.P. Angotti, em: Anais do IX Encontro Nacional de Pesquisa em Ensino de Física (Jaboticatubas, 2004).

[2] P.F.T. Dorneles, I.S. Araujo e E.A. Veit, Revista Brasileira de Ensino de Física 28, 487 (2006).

[3] S.J. Chapman, Fundamentos de Máquinas Elétricas (AMGH, Porto Alegre, 2013), $5^{\mathrm{a}}$ ed., v. 1, p. 66.

[4] S.D. Umans, Máquinas Elétricas (AMGH, Porto Alegre, 2014), $7^{\text {a }}$ ed., v. 1, p. 63. 
[5] https://www.electronica-pt.com/transformadores acessado em 01/11/2021.

[6] H.D. Young e R.A. Freedman, Física III - Eletromagnetismo (Pearson - Addison Wesley, São Paulo, 2009), 12 a ed., v. 3, p. 365.

[7] S.D. Umans, Máquinas Elétricas (AMGH, Porto Alegre, 2014), $7^{\mathrm{a}}$ ed., v. 1, p. 74.

[8] J.W. Nilsson e S.A. Riedel, Circuitos Elétricos (Pearson Prentice Hall, São Paulo, 2009), 8 a ed., v. 1, p. 234.

[9] T.C.S. Lopes e V.P.B. Aguiar, Projeto e Análise de Parâmetros de Operação de um Autotransformador. Trabalho de Conclusão de Curso, Universidade Federal Rural do Semi-Árido, Mossoró (2018). 